\title{
The language of genetics
}

\author{
The language used in genetic and medical research to describe populations has a fraught history, and current \\ practices must be sensitively considered when reporting on genetic cohorts and analyses.
}

T:

he language used to describe individuals or groups in genetics research can be imprecise, confusing or even offensive. As a global community, geneticists need to take a broad perspective when selecting what language to use.

Journals can have a large influence on the field in terms of implementing policies towards more reproducible, ethical and equitable research. Our editorial policies provide oversight to ensure that research is conducted responsibly. Given the hideous racist history of the genetics field, we have to work actively to shape policies that prevent a repetition or continuation of abhorrent or objectionable practices. To do this, we need to involve different stakeholders and communities, particularly those that have been the most vulnerable to abuse and being marginalized.

Specifically, regarding the language used to describe genetic cohorts and analyses, we need maximum community input when we are making policies and recommending best practices.

In this issue of Nature Genetics, we publish a Comment on the misuse of the term 'trans-ethnic' in genomics research. Mireille Kamariza, Lorin Crawford, David Jones and Hilary Finucane argue that using trans-ethnic as a shorthand to describe genetic cohorts or genome-wide association studies is inaccurate and can lead to confusion. The authors recommend that this term be avoided and that alternatives, such as multi (or cross)-population or multi (or cross)-ancestry, be used instead as appropriate. They urge geneticists to use descriptions that are as accurate as possible when discussing study participants. We endorse these recommendations as best practice and encourage authors to carefully consider the language that they use when writing manuscripts and presenting data.

In addition, the authors recommend that geneticists come together with colleagues from other fields, such as sociology and anthropology, to work towards arriving at agreed upon terms to use in genetics research when describing populations. We in turn agree with this proposal, and encourage the community to continue forwarding these discussions, with global representation, to ensure participation and leadership from researchers.

As such, we are putting out a call for input on the language used in genetics research, in the form of Commentary articles. We aim to represent a global genetics community and thus we particularly encourage contributions from underrepresented voices. No particular group should have a monopoly on determining what are the best terms to use in genetics research. We ask that the commentaries be clear, concise and field-forwarding. Comment articles are peer-reviewed. Recommendations should be justified and relatively easy to implement (although we acknowledge that there are profound complexities that prevent simple solutions in every case).
We of course recognize that that there are many people having these conversations and that these discussions have been going on for a long time. There are multiple resources available - for example, from TOPMed - that include guidelines for reporting race, ethnicity and ancestry. However, as was recently highlighted, merely changing terms will not fix the issues of racism in genetics research that the use of sensitive language seeks to address. We need to take a broader approach that prioritizes building partnerships across disciplines and maximizes global input, especially from groups that have been underrepresented in genetics research.

Coming to agreement on consensus terms used to describe genetic cohorts or analyses is complicated. In addition, the process of arriving at such terms must encompass acknowledgment of past harms, and involvement and leadership from members of those groups who have been subjected to those harms. In the meantime, using language that is as accurate and sensitive as possible is required, and editors, authors, reviewers and readers should be aware of this.

Please get in touch with us if you would like to discuss submitting a commentary article on language used in genetics research.

Published online: 5 November 2021 https://doi.org/10.1038/s41588-021-00965-1 